\title{
Clinical and Pathological Features of Lipoleiomyoma of the Uterine Corpus: A Review of 76 Cases
}

\author{
Metin Akbulut, Mehmet Gündoğan, Aygün Yörükoğlu
}

Department of Pathology, Pamukkale University Faculty of Medicine, Denizli, Turkey

\begin{abstract}
Background: Uterine lipoleiomyoma is a rare and specific type of leiomyoma with a considerable amount of adipocytes.

Aims: The aim of the study was to investigate the clinical, pathological and immunohistochemical features of lipoleiomyoma of the uterine corpus, and review its histogenesis and differential diagnosis from other neoplastic and non-neoplastic lesions in order to obtain a detailed profile of this somewhat uncommon lesion.

Study Design: Descriptive study.

Methods: This study is a retrospective analysis of 70 consecutive women with 76 lipoleiomyomas, who underwent surgery mainly for uterine leiomyoma and gynecological carcinomas between January 2000 and April 2013. Clinical and pathological information was obtained from medical records. Immunohistochemistry was applied in selected cases. Parametric methods were used to compare clinical and pathologic features.
\end{abstract}

Results: The patients ranged in age from 34 to 77 years (mean 55.49 years). Lipoleiomyomas ranged from 0.5 to $55 \mathrm{~cm}$ in diameter (mean $5.50 \mathrm{~cm}$ ). Typical macroscopic and microscopic features were noted.
Sixty-nine $(90.7 \%)$ tumors were in the uterine corpus and five $(6.5 \%)$ were in the cervix. One broad ligament tumor and one retroperitoneal tumor were also studied. No tumors displayed cytologic atypia, mitosis, necrosis, calcification, or other degenerative changes. Immunohistochemically, the adipose tissue element was positive for vimentin, desmin, S100 protein, estrogen (ER), progesterone (PR), and Ki-67. Among patients with lipoleiomyomas, 53 cases $(75.7 \%)$ had different types of lesions associated with hyperestrogenic status, such as adenomyosis, endometriosis, endometrial hyperplasia, and polyps, complex atypical endometrial hyperplasia, and gynecologic carcinomas. The follow-up period ranged from one to eight years (mean 4.6 years). There were no recurrences or tumor-related fatalities.

Conclusion: In the present study, the lipoleiomyomas were seen more frequently in patients with adenomyosis, endometriosis, endometrial hyperplasia, polyps, and various gynecologic carcinomas. Lipoleiomyoma of the uterus seems to have a benign clinical course. Balkan Med J 2014;31:224-9).

Key Words: Estrogen, gynecologic carcinoma, histogenesis, lipoleiomyoma
Lipoleiomyoma is a rare and easily recognized benign variant of uterine leiomyoma, composed of an intimate admixture of mature smooth muscle cells and adipocytes, often with the former element predominant, most commonly found in the uterine corpus (1-4).

In 1916, Lopstein first described a lipoleiomyoma, but this received little attention in the literature (5). Its lack of specific histological features led researchers to underestimate its occurrence rate. The largest reports in the literature belong to Wang et al. (1) with 50 cases and Aung et al. (2) with 17 cases. To this day, there is no uniform accepted classification and the histogenesis is not resolved. Willen and Pounder $(5,6)$ designated these tumors as "uterine fatty tumors" and subdivided them into "lipoma" and "mixed lipoma/leiomyoma" (lipoleiomyoma). Modern research rejects the view that these tumors are hamartomas, choristomas, fatty metamorphosis, or lipomatous degeneration. They probably represent tumor metaplasia within a leiomyoma. However, some studies show monoclonality (7), and lipoleiomyoma is now regarded as a distinctive true neoplasm (8). Therefore, its pathogenesis and clinical significance remain to be clarified. Regardless of its origin, lipoleiomyomas have been consistently considered benign.

Our study aimed to investigate the clinical, pathological and immunohistochemical features of uterine lipoleiomyoma and to confirm the benign nature of these tumors.

\section{MATERIAL AND METHODS}

This is a retrospective study of 70 patients who had undergone hysterectomy and myomectomy over a 13-year period 
(January 2000 - April 2013). Seventy patients showed 76 lipoleiomyomas in the Department of Pathology.

For routine histological analysis, we fixed resected specimens in $10 \%$ formalin, embedded them in paraffin and stained them with hematoxylin and eosin (H\&E). Pathological examination of the specimens was performed in the same hospital in the Department of Pathology. A single pathologist (MA) reviewed and evaluated all the slides on a retrospective basis with a light microscope. Relevant clinical, pathological, and radiological information was obtained from the patient's files and record forms. In the follow-up, we reviewed medical records and communicated with the patients.

Histological and pathological information was collected, including the presence and degree of accompanying carcinoma, endometrial hyperplasia, endometrial polyp, endometriosis, adenomyosis, atypia of smooth muscle cells, atypia of adipocytes, necrosis, mitosis, lymphocytic infiltration, and tumor borders (circumscribed versus infiltrative). The presence and amount of adipocytes was graded using a three tier grading system as Grade 1: minimal, focal; Grade 2: minimal to moderate, multifocal; Grade 3: abundant, multifocal, evenly distributed.

The histological diagnosis used H\&E stains. Immunohistochemical (IHC) staining was previously performed in many cases on fixed paraffin-embedded sections on a Dako Autostainer Link48 (Dako North America, Inc., California, USA). We performed immunohistochemical analysis on 4-mm thick, formalin-fixed, paraffin-embedded representative tissue sections from selected cases, using an indirect immunoperoxidase technique and using antibodies against desmin, vimentin, smooth muscle actin (SMA), S100, HMB-45, Ki-67, ER, and PR receptors (DAKO, Glostrup, Denmark). We used appropriate positive controls for each antibody. Table 1 summarizes the monoclonal antibodies used.

The study was carried out in accordance with the Declaration of Helsinki and approved by the institutional ethical committee.

\section{Statistical analysis}

Findings were analyzed using the statistical software SPSS for Windows, version 17 (SPSS, Chicago, IL, USA). With the Spearman correlation and chi-square analysis, we tested for correlations of lipoleiomyomas with various pathologic and clinical features, including age, carcinoma, endometrial hyperplasia, endometrial polyps, endometriosis, and adenomyosis. A $p$ value of $<0.05$ was used to define a significant association.

\section{RESULTS}

From 2000 to 2013, the Pathology Department's files included 70 patients with 76 uterine lipoleiomyomas. During
TABLE 1. Antibodies used

\begin{tabular}{lccc}
\hline Antibody & Clone & Dilution & Manufacturer \\
\hline Desmin & D33 & $1 / 50$ & Thermo \\
Vimentin & V9 & Ready to use & Dako \\
SMA & 1A4 & Ready to use & Dako \\
S100 & Polyclonal & Ready to use & Dako \\
HMB-45 & HMB-45 & Ready to use & Dako \\
Ki-67 & SP6 & $1 / 200$ & Thermo \\
ER & EP1 & Ready to use & Dako \\
PR & PgR636 & Ready to use & Dako \\
\hline
\end{tabular}

SMA: smooth muscle actin; ER: estrogen; PR: progesterone

the same duration, 2600 uterine leiomyomas were seen, thus lipoleiomyoma of the uterus comprised $2.9 \%$ of all uterine leiomyomas at our institution.

The patients ranged in age from 34 to 77 years, with a mean age of 55.49 years, and with median age of 55.50 years. Fiftyeight patients $(82.8 \%)$ were postmenopausal. None were pregnant. Most sought medical attention for symptomatic leiomyomas (pelvic pain, enlarged uterus, and/or dysmenorrhea). The others sought treatment of a gynecological neoplasm.

The patients underwent a variety of different surgical procedures: $62(88.5 \%)$ underwent hysterectomy and bilateral salpingo-oophorectomy, while eight (11.4\%) underwent myomectomy. The majority of lipoleiomyomas were in the uterine corpus (69 cases, 90.7\%), but some were found in the cervix (5 cases, $6.5 \%)$. Another was retroperitoneal and one was located in the broad ligament (1.3\%). Fifty-eight tumors affected the uterine corpus, and were intramural, two were submucosal, and nine were subserosal. Six patients $(8.5 \%)$ had two nodules of lipoleiomyomas each. All others had solitary lipoleiomyomas.

The size of the lipoleiomyomas ranged from 0.5 to $55 \mathrm{~cm}$ in diameter, with a mean of $5.50 \mathrm{~cm}$. All were nodular and sharply circumscribed. Some were firm and rubbery, while others were fibro-fatty, with pale to yellow irregular soft areas. In cases with considerable amounts of fat, the cut surface was yellow (Figure 1). Grade I (minimal and concentrated in only focal areas) adipocytes were seen in $35(46.05 \%)$, grade II (moderate) in $3(3.9 \%)$ and grade III (abundant, multifocal and evenly distributed throughout the tumor) in $38(50 \%)$ lipoleiomyomas.

Lymphocytes massively infiltrated the lipoleiomyomas in six cases $(7.8 \%)$. The tumors were otherwise typical of uterine leiomyomas. None displayed mitosis, cytologic atypia (in smooth muscle cells or adipocytes), immature lipoblasts, necrosis, calcification, or other degenerative changes. The tumors were sharply separated from the adjacent myometrium, with no infiltrative margins. In seven cases $(9.2 \%)$, in addition to smooth muscle tissue and adipose tissue, angiomatous hyperplasia was also present. 
TABLE 2. Histological findings in patients with uterine lipoleiomyomas $(\mathrm{n}=70$ patients $)$

\begin{tabular}{lc}
\hline Histological lesions & Number of patients (\%) \\
\hline Cervical squamous intraepithelial lesion & $3(4.2 \%)$ \\
Ordinary leiomyoma & $33(47.1 \%)$ \\
Atypical leiomyoma & $1(1.4 \%)$ \\
Adenomyosis & $24(34.2 \%)$ \\
Endometriosis & $5(7.1 \%)$ \\
Endometrial polyp & $10(14.2 \%)$ \\
Uterus adenomatoid tumor & $2(2.8 \%)$ \\
Endometrial hyperplasia & $12(17.1 \%)$ \\
Endometrial atypical hyperplasia & $3(4.2 \%)$ \\
Endometrioid carcinoma & $7(10 \%)$ \\
Endometrial carcinosarcoma & $1(1.4 \%)$ \\
Ovarian teratoma & $1(1.4 \%)$ \\
Ovarian fibrothecoma & $2(2.8 \%)$ \\
Ovarian serous cystadenofibroma & $4(5.7 \%)$ \\
Ovarian serous carcinoma & $3(4.2 \%)$ \\
Breast carcinoma & $1(1.4 \%)$ \\
Gastric carcinoma & $1(1.4 \%)$ \\
Rectum carcinoma & $1(1.4 \%)$ \\
\hline
\end{tabular}

In all 70 patients, we found 12 cases (17.1\%) of gynecologic malignancies; including eight endometrial (11.4\%), three ovarian, $(4.2 \%)$ and one breast (1.4\%). In the patients with an endometrial malignancy, uterine lipoleiomyoma was an incidental diagnosis during surgery. In cases in which there was a malignancy, there was no gross or microscopic contiguity between the lipoleiomyoma and the malignancy. The present study recognized eight cases of diabetes mellitus and six cases of hypothyroidism. Table 2 summarizes the diagnoses other than lipoleiomyoma.

We found no significant correlation between amount of adipocytes and other clinical and pathological features, none between the proportion of lipomatous and leiomyomatous components and the size of tumor, and none between the amount and distribution of the lipomatous component and the age of the patients.

In the immunohistochemical investigation, we studied the expression of ER and PR receptors, Ki-67, SMA, HMB-45, desmin, vimentin in selected cases. The adipose tissue and smooth muscle elements were positive for vimentin, desmin, S100 protein, ER, PR, and Ki-67 (Figures 2 and 3). The examined tumor markers (CEA, CA19-9, CA125) were normal.

There were no recurrences or deaths attributed to the lipoleiomyomas during a mean follow-up period ranging from one to eight years (mean, 4.5 years). Follow-up data are available for 62 patients $(88.5 \%)$.

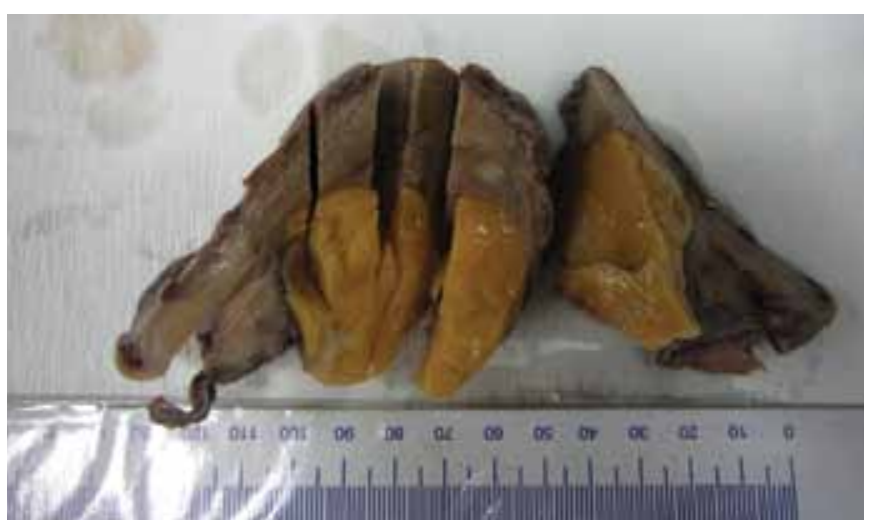

FIG. 1. Gross image of a lipoleiomyoma with a high adipocyte component, showing a well-circumscribed soft mass with a yellow cut surface in the uterine body

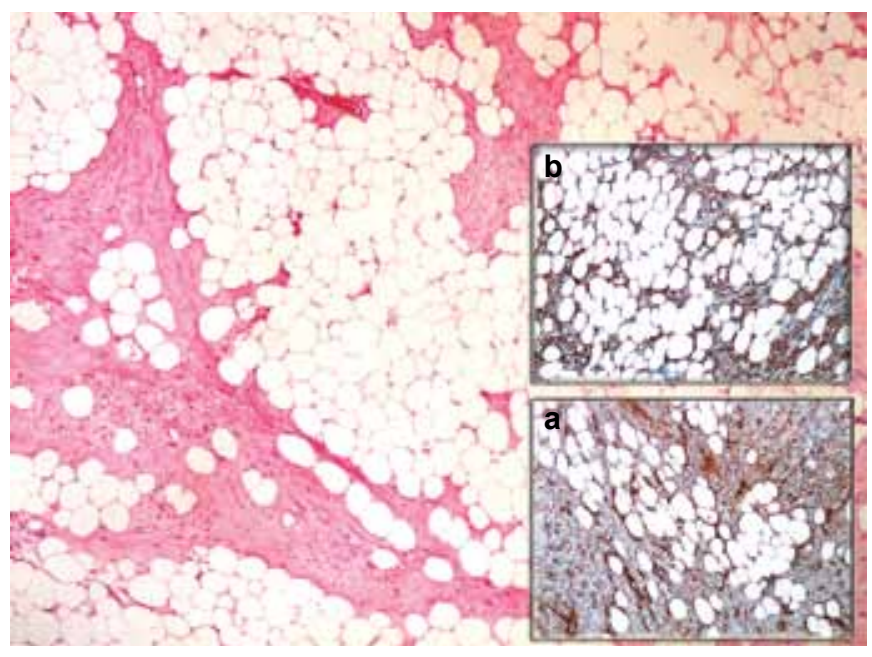

FIG. 2. a, b. Microscopic examination of a lipoleiomyoma with a high adipocyte component (H\&E, 40x). The adipose tissue is positive for vimentin (immunostaining 100x) (a), and for desmin (immunostaining 100x) (b)

\section{DISCUSSION}

In the present study, 53 patients with lipoleiomyomas (75.7\%) had different types of lesions associated with hyperestrogenic status such as adenomyosis, endometriosis, endometrial hyperplasia, polyps, complex atypical endometrial hyperplasia, and gynecologic carcinomas. We suggest estrogenic manifestations may be an important factor in the development of lipoleiomyomas.

Uterine lipoleiomyoma is a rare and specific type of leiomyoma with a considerable amount of adipocytes (1-3). The clinical features and histogenesis of this tumor continue to be uncertain. In most of our patients, lipoleiomyoma was incidentally found during surgery for other reasons. As observed in previous studies, lipoleiomyoma most commonly grows in the uterine corpus at the subserosal or intramural levels 


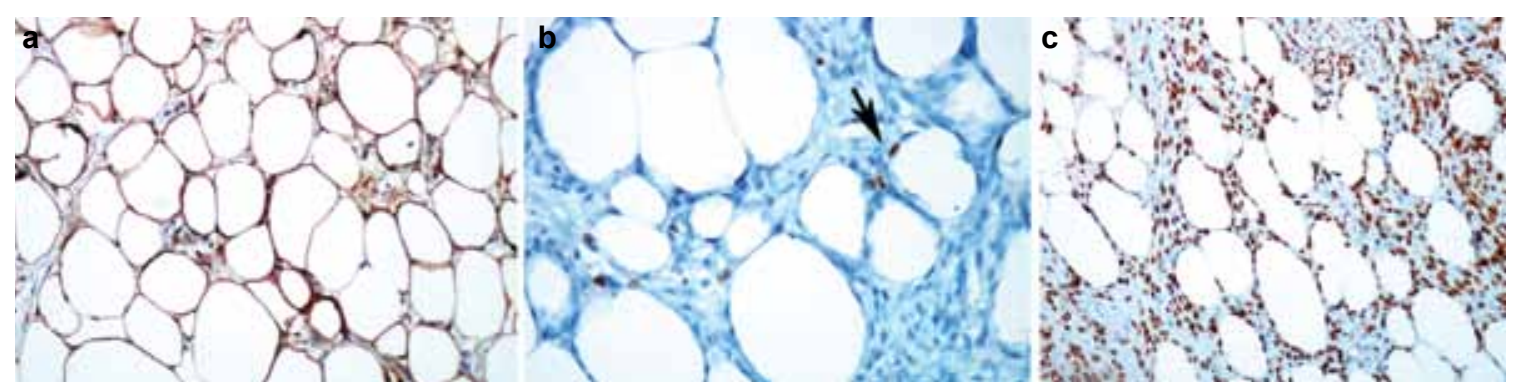

FIG 3. a-c. Lipoleiomyoma consisting of mature smooth muscle cells and adipose tissue positive for $\mathrm{S} 100$ protein (immunostaining 100x) (a). The adipose tissue is positive for Ki-67 (short arrow, immunostaining 400x) (b). The smooth muscle cells and adipose tissue are positive for estrogen receptors (immunostaining, 200x) (c)

$(1,2,6,9)$; however, it has been reported at other sites, including the cervix $(1,3,10-12)$ as well as in intraligamentary, peritoneal, and ovarian areas $(1,2,13)$.

Lipoleiomyoma represented $2.9 \%$ of all leiomyomas in our study; a slightly lower incidence $(0.03 \%$ to $2.1 \%)$ has been reported in the literature $(1,2,5,9)$. This may be related to the extensive sampling of all leiomyomatous nodules. Many lipoleiomyomas ranged from 0.5 to $2 \mathrm{~cm}$ in the study. The clinical presentation of uterine lipoleiomyomas is non-specific, and these tumors generally occur in asymptomatic obese perimenopausal or menopausal women $(1,2,6)$. The most common signs and symptoms are abnormal uterine bleeding, abdominal and/or pelvic pain, a palpable mass, urinary frequency, incontinence and a sensation of pressure similar to those of typical leiomyomas $(1,2)$. Although most leiomyomas tend to predominate in women of reproductive age, lipoleiomyomas are frequently seen in older women. The mean ages of the patients in the series of Wang et al. (1) and Aung et al. (2) were 53.9 and 59.9 years, respectively. Lipoleiomyomas may rarely be multiple, but often are single, with variations in size (14). In our study, six patients had two nodules of lipoleiomyoma. More than two lipoleiomyomas have not been described in the literature. Lipoleiomyomas range from 0.5 to $55 \mathrm{~cm}$ in size, with a mean of $5.50 \mathrm{~cm}$. Four were larger than $10 \mathrm{~cm}$. The literature contains reports of large lipoleiomyomas $(4,6,10,11)$.

Several pathogenic mechanisms may underlie the presence of adipocytes within leiomyomas, including misplaced embryonic fat cells, metaplasia of muscle, or differentiation of connective tissue cells into fat cells, lipocytic differentiation of a specific primitive connective tissue cell, perivascular fat cells accompanying the blood vessels into the uterus, inclusion of fat cells in the uterine wall during surgery, or fatty infiltration or degeneration of connective tissue $(5,15)$. Fukunaga suggests that at least some lipoleiomyomas result from lipomatous metaplasia of leiomyomas, a theory supported by immunohistochemical findings (14). However, numerous immunohistochemical studies have indicated a complex histogenesis of lipoleiomyoma which might arise from multipo- tential undifferentiated mesenchymal cells (15) or from direct transformation of smooth muscle cells into adipocytes (16). Aung et al. (2) and Terada show focal proliferation of adipocytes, labeled by Ki-67 staining, and further suggest that the adipocyte component is neoplastic rather than degenerative (10). Terada also showed the presence of ER and PR in the adipose element, and suggested the adipose component of lipoleiomyoma is specific fat tissue related to the female genital organs (10). In our study, fat tissue was positive for Ki-67, desmin, vimentin, and ER and PR receptors, supporting the direct transformation of muscle cells into adipose cells that may originate from the transformation of a totipotent mesenchymal cell $(3,17)$.

Uterine smooth muscle cells have divergent differentiation potential and may undergo many alterations, including differentiation toward adipocytes or skeletal muscle. We suggest that the histological structure and immunohistochemical profile suggest that the lipoleiomyomas probably result from a metaplasia of the smooth muscle cells more than a true neoplastic process. Many authors have performed cytogenetic studies of uterine lipoleiomyomas, and suggest that uterine lipoleiomyomas have a pathogenic origin similar to that of typical leiomyomas (7). Cytogenetic studies might therefore clarify the origin of this tumor. In the literature, many authors provide evidence to support a hyperestrogenic state contributing to the development of lipoleiomyomas $(10,18,19)$. Lipoleiomyomas may also be associated with some metabolic disorders such as hyperlipidemia, hypothyroidism, and diabetes mellitus $(4,10)$. After menopausal changes, lipid metabolism may play a role in lipoleiomyoma development. Adipocyte differentiation in the myometrium has been observed under conditions such as toxemia during pregnancy (20), so environmental factors in the uterus may play a role in adipocyte differentiation in lipoleiomyomas $(18,20)$. The relationship between gynecologic malignancies, which may originate from the uterus, cervix, or ovaries, and coexistent lipoleiomyoma has been reported in only a few studies $(1-3,6,21)$. In two large studies, Aung et al. (2) reported two (11\%) gynecologic carcinomas in 17 cases and Wang et al. (1) reported 
10 cases $(20 \%)$ of patients with uterine lipoleiomyoma associated with gynecologic malignancies. In our 70 patients, we found 12 cases (17.1\%) of gynecologic malignancies: eight endometrial, three ovarian, and one breast. In these patients, a concomitant gynecologic malignancy and estrogen-related lesions appeared to be common, presumably related to estrogenic stimulation in these patients. This observation suggests that finding adipocytes in an otherwise normal leiomyoma should lead to further detailed clinical and pathological evaluation to search for the presence of a coexistent gynecologic neoplasm. This was also a significant observation of the present study. However, further studies on larger populations are needed to establish any association with malignancy, estrogenic status or metabolic disorders.

Recognition of this rare and benign tumor is of great importance, because it may be confused with other gynecologic maladies, including spindle cell lipoma, angiolipoma, angiomyolipoma, atypical lipoma, myelolipoma, myxoid mesenchymal tumors, pelvic fibromatosis, well-differentiated liposarcoma, carcinosarcoma (malignant mixed Müllerian tumor) with heterologous liposarcomatous differentiation, ovarian fatty tumors, and benign or malignant degeneration of ordinary leiomyomas $(1,2)$. Lipoleiomyosarcomas arising in uterine lipoleiomyomas have also been reported $(22,23)$. Intravenous lipoleiomyomatosis has been rarely reported (24). One report of intravascular lipoleiomyomatosis shows extension of the tumor into the inferior vena cava, a potentially life-threatening effect. While lipoleiomyomas are considered benign, close patient follow-up is necessary.

Lipoleiomyomas, if asymptomatic, require no treatment and are clinically similar to leiomyomas, so it is essential to distinguish these tumors from other tumors that need surgical excision (1). Uterine lipoleiomyomas are often diagnosed preoperatively as uterine leiomyomas or mature ovarian teratomas (25). Fat within a uterine mass can be diagnostic of lipoleiomyoma or pure lipoma. Although ultrasonography has been thought to be specific enough to allow for tissue description and to establish a precise preoperative diagnosis (26), magnetic resonance imaging (MRI) or computed tomography (CT) can exclusively show the fat content within the tumor (27). Therefore, in combination with sonography, CT and MRI may assist in the preoperative diagnosis of lipoleiomyomas $(1,9,16,25,26)$. However; differentiation cannot be made on the basis of imaging findings alone, and histopathological evaluation must be performed to distinguish lesions in the differential diagnosis of lipoleiomyoma $(9,21)$.

In conclusion, uterine lipoleiomyomas are benign uterine lesions that, in the present study, accounted for $2.9 \%$ of benign uterine leiomyomas. We conclude that uterine lipoleiomyomas represent a variant of leiomyoma with adipocyte differentiation rather than a degenerative or neoplastic change in ordinary leiomyoma. Patients with lipoleiomyomas have many estrogen-related conditions including endometriosis, adenomyosis, endometrial hyperplasia, polyps, and gynecologic malignancies. In this study, based on the follow-up information, lipoleiomyomas showed a favorable outcome. However, the pathogenesis and clinical significance of these rare neoplasms remain to be clarified.

Ethics Committee Approval: Ethics committee approval was received for this study from ethics committee of Pamukkale University Faculty of Medicine (decision no: 60116787/020/20462 2013/06).

Informed Consent: N/A.

Peer-review: Externally peer-reviewed.

Author contributions: Concept - M.A.; Design - M.A., M.G.; Supervision - M.A., M.G., A.Y.; Resource - M.A., M.G., A.Y.; Materials - M.A., A.Y.; Data Collection\&/or Processing - M.A., A.Y.; Analysis\&/or Interpretation M.A., A.Y.; Literature Search - M.A., A.Y.; Writing - M.A.; Critical Reviews - M.A.

Conflict of Interest: No conflict of interest was declared by the authors.

Financial Disclosure: The authors declared that this study has received no financial support.

\section{REFERENCES}

1. Wang X, Kumar D, Seidman JD. Uterine lipoleiomyomas: A clinicopathologic study of 50 cases. Int J Gynecol Pathol 2006;25:239-42. [CrossRef]

2. Aung T, Goto M, Nomoto M, Kitajima S, Douchi T, Yoshinaga M, et al. Uterine lipoleiomyoma: A histopathological review of 17 cases. Pathol Int 2004;54:751-8. [CrossRef]

3. Shintaku M. Lipoleiomyomatous tumors of the uterus: A heterogeneous group? Histopathological study of five cases. Pathol Int 1996;46:498-502. [CrossRef]

4. Akbulut M, Soysal ME, Duzcan SE. Giant lipoleiomyoma of the uterine corpus. Arch Gynecol Obstet 2008;278:291-3. [CrossRef]

5. Willen R, Gad A, Willen H. Lipomatous lesions of the uterus. Virchows Arch A Pathol Anat Histopathol 1978;377:351-61. [CrossRef]

6. Pounder DJ. Fatty tumours of the uterus. J Clin Pathol 1982;35:1380-3. [CrossRef]

7. Havel G, Wedell B, Dahlenfor R, Mark J. Cytogenetic relationship between uterine lipoleiomyomas and typical leiomyomas. Virchows Archiv B Cell Pathol 1989;57:77-9. [CrossRef]

8. Sudhamani S, Agrawal D, Pandit A, Kiri V M. Lipoleiomyoma of uterus: A case report with review of literature. Indian J Pathol Microbiol 2010;53:840-1. [CrossRef]

9. Prieto A, Crespo C, Pardo A, Docal I, Calzada J, Alonso P. Uterine lipoleiomyomas: US and CT findings. Abdom Imaging 2000;25:655-7. [CrossRef]

10. Terada T. Giant subserosal lipoleiomyomas of the uterine cervix and corpus: A report of 2 cases. Appl Immunohistochem Mol Morphol 2012. [Epub ahead of print] [CrossRef]

11. Terada T. Huge lipoleiomyoma of the uterine cervix. Arch Gynecol Obstet 2011;283:1169-71. [CrossRef]

12. Volpe R, Canzonieri V, Gloghini A, Carbone A. Lipoleiomyoma with metaplastic cartilage (benign mesenchymoma) of the uterine cervix. Pathol Res Pract 1992;188:799-803. [CrossRef]

13. Schindl M, Birner P, Losch A, Breitenecker G, Joura EA. Preperitoneal lipoleiomyoma of the abdominal wall in a postmenopausal woman. $M a-$ turitas 2000;37:33-6. [CrossRef] 
14. Fukunaga M. Benign "metastasizing" lipoleiomyoma of the uterus. Int $J$ Gynecol Pathol 2003; 22:202-4. [CrossRef]

15. Sieinski W. Lipomatous neometaplasia of the uterus: report of 11 cases with discussion of histogenesis and pathogenesis. Int J Gynecol Pathol 1989;8:357-63. [CrossRef]

16. Resta L, Maiorano E, Piscitelli D, Botticella MA. Lipomatous tumors of the uterus. Clinico-pathological features of 10 cases with immunocytochemical study of histogenesis. Pathol Res Pract 1994;190:378-83. [CrossRef]

17. Bolat F, Kayaselçuk F, Canpolat T, Erkanlı S, Tuncer I. Histogenesis of lipomatous component in uterine lipoleiomyomas. Turk Patoloji Derg 2007;23:82-6.

18. Schindl M, Birner P, Losch A, Breitenecker G, Joura EA. Preperitoneal lipoleiomyoma of the abdominal wall in a postmenopausal woman. $M a$ turitas 2000;37:3-6. [CrossRef]

19. Akpolat I, Sertcelik A, Cömert S, Bulay O, Ortac F. ERRP-29 and ER staining in uterine lipoma and lipoleiomyoma. Acta Oncol 1996;35:108. [CrossRef]

20. Haust MD, Las Heras J, Harding PG. Fat-containing uterine smooth muscle cells in "toxemia": possible relevance to atherosclerosis? Science 1977;195:1353-4. [CrossRef]
21. Jouini S, Tawfik A, Alewa A, Wachuku E, Ayadi K. What's your diagnosis? Subserosal uterine lipoleiomyoma fortuitously associated with endometrial carcinoma. Ann Saudi Med 2001;21:251-4.

22. Lee HP, Tseng HH, Hsieh PP, Shih TF. Uterine lipoleiomyosarcoma: report of 2 cases and review of the literature. Int J Gynecol Pathol 2012;31:358-63. [CrossRef]

23. McDonald AG, Dal Cin P, Ganguly A, Campbell S, Imai Y, Rosenberg AE, Oliva E, et al. Liposarcoma arising in uterine lipoleiomyoma: a report of 3 cases and review of the literature. Am J Surg Pathol 2011;35:221-7. [CrossRef]

24. Vural C, Özen Ö, Demirhan B. Intravenous lipoleiomyomatosis of uterus with cardiac extension: A case report. Pathol Res Pract 2011;207:131-4. [CrossRef]

25. Su WH, Wang PH, Chang SP, Su MC. Preoperational diagnosis of a uterine lipoleiomyoma using ultrasound and computed tomography images: A case report. Eur J Gynaecol Oncol 2001;22:439-40.

26. Pham CA, Atri M, Senterman MK. Ultrasonographic appearance of uterine lipoleiomyoma. Can Assoc Radiol J 1993;44:463-5.

27. Tsushima Y, Kita T, Yamamoto K. Uterine lipoleiomyoma: MRI, CT and ultrasonographic findings. Br J Radiol 1997;70:1068-70. [CrossRef] 